\title{
ANALYSIS OF ASSIGNMENT RULES IN A MANUALLY OPERATED DISTRIBUTION WAREHOUSE
}

\author{
Uwe Clausen \\ Peiman Dabidian \\ Daniel Diekmann \\ Ina Goedicke \\ Moritz Pöting \\ TU Dortmund University \\ Institute of Transport Logistics \\ Leonhard-Euler-Str. 2 \\ D-44227 Dortmund, GERMANY
}

\begin{abstract}
Due to strong market competition, operators of production and logistics systems are constantly looking for measures to increase the efficiency of internal handling and storage processes. The scope of this application-oriented paper will be a manually operated distribution warehouse with a capacity of more than 40,000 pallets connected to an order-picking and a value-added-service area. Different concepts to control the warehouse, such as storage location assignment strategies or the control of the forklift fleet, are investigated with regard to their ability to improve the handling performance in real world applications. Therefore, during the modeling process several relevant practical characteristics e.g., traffic obstruction of forklifts in storage aisles during loading and unloading activities, dimensions of storage locations or storage and retrieval strategies, are taken into account. The results show that concepts can significantly improve warehouse operations.
\end{abstract}

\section{INTRODUCTION}

Third-party logistics providers are specialized in managing a wide range of services for some or all supply chain functions of their customers. These services usually include warehousing and transport operations that are individually customized to meet the clients' requirement. In addition, several value-addedservices are integrated into the supply chain tailored to the customer demands and their delivery and service requirements. The increasing demand of integrated logistics services leads to a considerable growth of contract logistics in recent years. According to a study of Transport Intelligence, the global contract logistics grew in 2011 by $4.5 \%$ (Transport Intelligence 2012). It is one of the largest logistics market segment e.g., with more than one third market share in Germany, and achieves the highest growth rates (Kille and Schwemmer 2012). However, the market competition and the pressure on prices forces companies to continuously improve their operational performance. Since the relationship is usually based on significant volume and long-term contracts, typically between 3 to 5 years, logistics service providers are put in the position to successfully initiate re-optimization and achieve competitive advantages.

The content of this application-oriented paper is a manually operated distribution warehouse. Different activities and value-added-services are carried out in the warehouse. The spatial concentration of several services in one warehouse is a typical procedure, since this approach enables the logistics service provider to realize synergies and scale effects. However, this leads to a higher process complexity and requires intelligent solutions to plan and control the warehouse. The objective of this paper is the 


\section{Clausen, Dabidian, Diekmann, Goedicke, and Pöting}

examination of the performance of the forklift fleet with regard to changes in storage location assignment and forklift fleet control. Due to the considerably varying system load and the interdependency of parallel processes a discrete event simulation model is used to investigate handling performance.

\section{SYSTEM DESCRIPTION}

The examined warehouse provides an inter-regional distribution warehouse with a full-pallet rack storage system. It has line-by-line arranged bearing blocks. The storage area is spread over several adjacent buildings that provide a continuous main aisle. The stored items are divided with regard to several storage zones, categorized by weight and product groups, e.g. hazardous goods. In addition, there is a valueadded-service area where customized services are operated. The warehouse covers a storage space of approximately $30,000 \mathrm{~m}^{2}$ and an additional value-added-service area of more than $5,000 \mathrm{~m}^{2}$. The capacity of the full-pallet storage area contains 40,000 pallets with storage bays for single and double stacked pallets.

Besides the zoning, the storage strategy considers categories of A, B and C items, which are arranged by cross-aisle storage. The job scheduling is implemented as a central order planning disposition with mobile terminals. The supply with incoming items is done via a central incoming goods area. For dangerous goods there is a separate entrance area inside the hazardous area. Outgoing items are provided at the buffer areas of the outbound gates. Depending on operating time and working shifts up to 15 forklifts serve the racking storage. They are grouped into three different teams (inbound, outbound and value-added-service). Figure 1 depicts the layout and the main transport movements, that are performed by the different forklift teams.

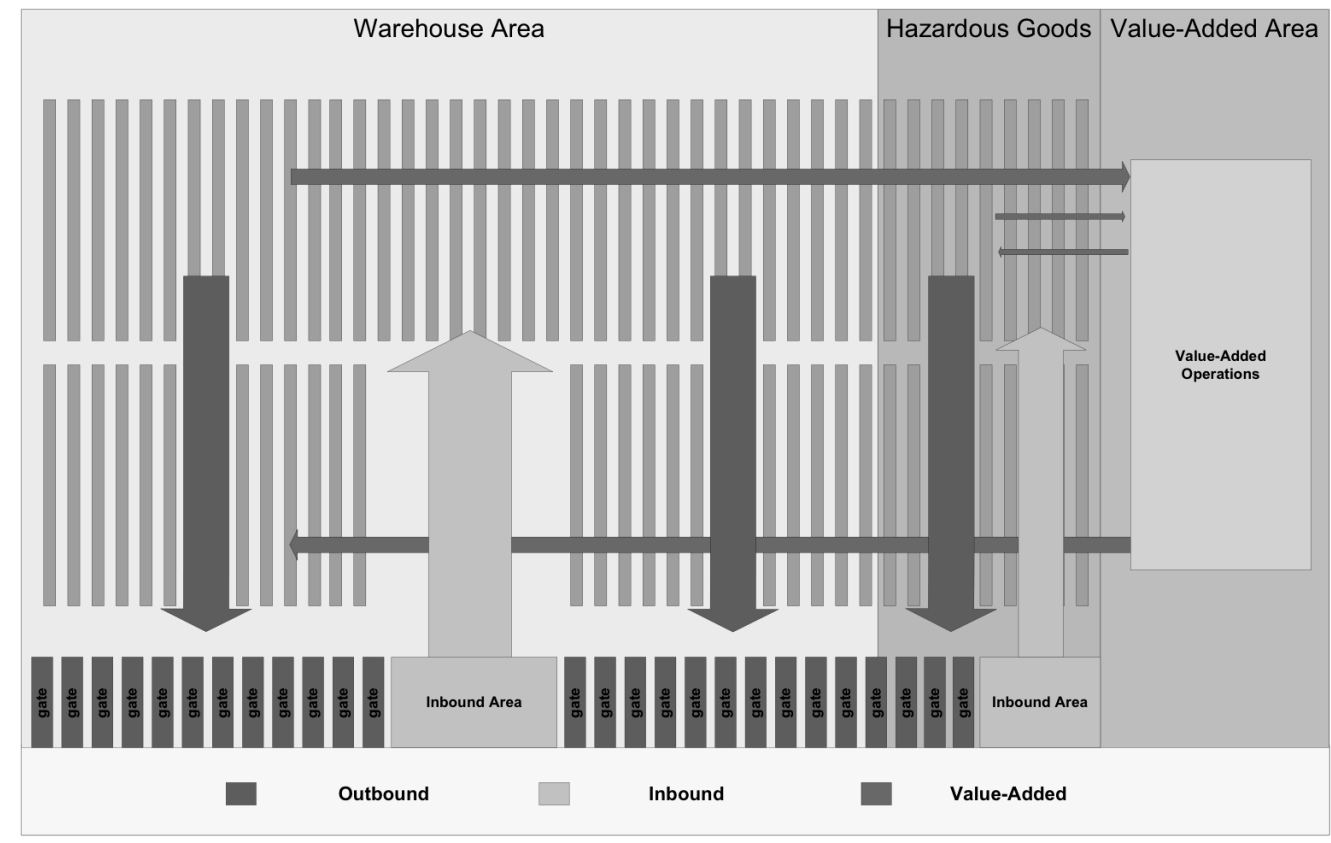

Figure 1: Layout of warehouse and transport movements.

Focusing on the handling performance of the forklift fleet, the operating cycle of a transport task consists of several activities that are described in Figure 2. After being triggered by a transport task, the forklift begins with an unloaded run to the designated position. On the way to the loading position the forklift can be interrupted by other forklifts (e.g., by an unloading or loading process within the narrow aisles). This interval of waiting is called blocking time and is considered separately. It most likely occurs, when several tasks are assigned with similar product types of incoming goods, causing a bay assignment directly abreast. 
Clausen, Dabidian, Diekmann, Goedicke, and Pöting

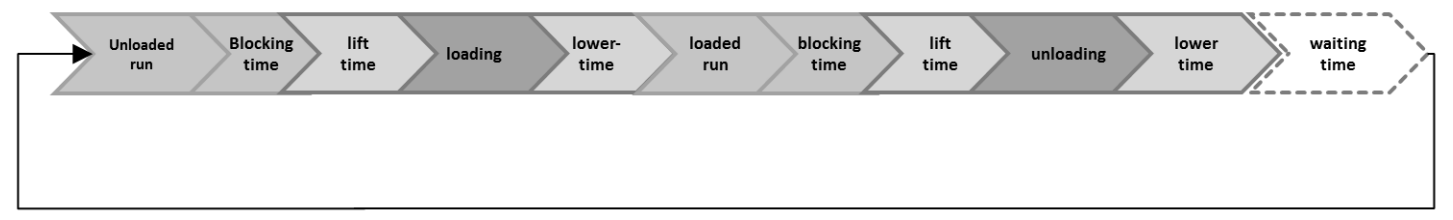

Figure 2: Operating cycle for a transport task.

When a forklift has reached its destination, the loading process begins, as the load gets picked at the inbound area, the value-added-area or the storage system. Picking processes from upper level warehouse locations include additional lift- and lower-times. After picking the goods, a loaded run to the target position is carried out. According to the unloaded run, blocking might temporary interrupt this process.

At the designated target destination, the process ends with unloading which can be considered analogous to the loading process. Thereby it has to be distinguished between inbound, outbound or replenishment tasks since the lift and lowering times only occur at upper level warehouse retrievals. Incoming goods have to be lifted when unloaded, outgoing and replenishment tasks have to be taken out of the warehouse when loaded. Once the process is finished, the forklift driver receives a new transport task from the warehouse management system via his mobile terminal. If there are no further tasks available, the forklift remains idle until being triggered by the next transport task.

In order to maximize the handling performance, it is the objective to minimize the overall average operating cycle.

\section{RELATED WORKS}

Around $75 \%$ of the warehouses are manually served by forklifts (Chan 2002). However, despite their dominance in practice, manually operated systems receive significantly less attention in research than full- or partly automated systems (de Koster et al. 2007). Takakuwa points out, that modeling large-scale non-automated distribution warehouses with forklifts is much more difficult in comparison to AS/RSsystems due to their complexity (Takakuwa et al. 2000).

According to the objective and the operating cycle (see Figure 2), related works are categorized into three improvement fields. First, aspects with regard to the loaded run are shown, then related works in relation to the unloaded run are described, and finally, blocking effects are discussed.

The loaded run is the main part of the transportation process and strongly depends on layout and design decisions. On strategic level common decisions are e.g., size and dimension of storage zones, (un)loading door location or aisle orientation. On tactical or operational level, decisions include e.g., grouping aisles into work zones or assigning products to storage locations. Levels are strongly independent and including them all in one model is intractable. Therefore researchers have to limit their studies on a few decision areas simultaneously (de Koster et al. 2007). Considering the operational level, storage location assignment strategies have a significant impact on material handling performance. The three popularly used storage location assignment strategies are random, dedicated and class-based storage. All strategies have their advantages and disadvantages, however the class-based storage is considered to be the most interesting one, as it provides advantages from dedicated storage, such as short distance for fast-moving items, and the random storage, such as the flexibility (Gu et al. 2007).

The unloaded run of a forklift depends on the dispatching strategy and the assignment of transport tasks. Koo and Jang divide dispatching decisions into two main cases (Koo and Jang 2002). In the first case, one or more vehicles are idle and immediately available when a transport task is sent to the dispatching system. This type of assignment rules is called task-initiated or machine-initiated rules and are more likely triggered when a lower vehicle utilization occurs (Egbelu and Tanchoco 1984). In the second case, a dispatching decision has to be made when a forklift drops off a load and is ready for the next task. This type of assignment rules is called vehicle-initiated rules and occur primarily in the given system due to the high forklift utilization. 
Clausen, Dabidian, Diekmann, Goedicke, and Pöting

By applying distance-based dispatching rules, such as shortest-travel-time-(distance)-first (STTF), the transporter is send to the closest load to be transported. These rules reduce the empty travel time, but are sensitive to the layout and the load locations in the system (de Koster et al. 2004). Especially in systems with high vehicle utilization, transport tasks in remote areas tend to have unacceptable high waiting times. In order to overcome this shortcoming strategies are used, which take into account additional parameters, such as the waiting time. These strategies are called multi-attribute strategies and in general outperform single-attribute strategies (Klein and Kim 1996, Le-Anh and de Koster 2006).

Blocking situations in manually operated warehouses occur whenever activities of a forklift are interrupted by other forklifts. According to Furmans et al. only few papers dealing with manually operated systems consider blocking (Furmans et al. 2009). The blocking situation in manual order picking systems can be divided into blocking situations in aisles and cross-aisles or at the base station. The potential of blocking rises with the number of forklifts. Additionally, the implementation of a class-based storage strategy increases the risk of blocking. The danger of aisle congestion increases especially in segments with high-volume (Petersen and Aase 2004).

\section{MODELING}

To analyze the interaction and effects of strategic planning as well as operational decisions a simulation suite was developed based on the simulation software Enterprise Dynamics by Incontrol Simulation Solutions. It permits the modeling and simulation of material and information flows in logistics nodes as explained in Deymann and Neumann (2008), and Clausen et al. (2012). Within the simulation suite modeling of the moved objects and resources takes place on a microscopical level. Furthermore, all handling and controlling processes of the warehouse can be modeled and parameterized.

In general, three types of data are necessary for modeling which have to be gathered about the real system (VDI Guideline 3633 Part 1):

- Technical data describes the topology and structure of the system. It includes information on the warehouse structure e.g., layout, equipment, capacities, process times.

- Organizational data describes the process structure and other organizational aspects, such as scheduling rules for working time, allocation of resources to tasks as well as other rules and restrictions of handling operations.

- System load data which describes the amount of handled pallets concerning time and volume aspects.

To implement a warehouse model, the gathered information has to be transferred into the required form. This results in various simulation objects that belong to the above mentioned groups. The group of technical data can be divided into two categories: static and dynamic simulation objects. Static objects form the layout of the system and are the basic elements for movements of the dynamic objects. The warehouse layout is modeled to scale with the help of warehouse modules considering each storage location within the shelves and the different shelf heights which define the suitability for single or double stacked pallets. Furthermore, buffer areas for incoming and outgoing pallets and the picking areas belong to the static simulation objects and are modeled as queues. They also form the system boundaries as they are the sources and sinks of the pallet movements. Additionally, the network for the forklifts is mapped as a network of nodes and bidirectional connections between them. This network is based on the actual distances. The forklifts are routed on the shortest path in the transport network based on a Dijkstra algorithm. The category of technical data contains the dynamic simulation objects which move within the system: pallets and forklifts. Each pallet is modeled by a single object with all relevant attributes, such as item type, height, weight, ability to double stack or item expiry. Furthermore, the amount of forklifts used in the real system is integrated into the model. The relevant process times for pallet handling are determined based on an extensive process study done by the warehouse operating company: lift time and lower time, loading time, unloading time. Furthermore, the network configuration for internal transports is modeled in a specific way to allow the consideration of blocking effects between different forklifts loading or unloading in one aisle. It is divided into sections which correspond with the dimension of a 


\section{Clausen, Dabidian, Diekmann, Goedicke, and Pöting}

warehouse module. Therefore, it is possible to block the specific route section in an aisle when placing or picking a pallet at the warehouse module takes place. Two different types of transport sections are distinguished. Main aisles are always open and can be used by several forklifts because aisle width is sufficient for forklifts passing each other. Aisles between warehouse objects are narrower and are therefore blocked section-wise during the picking or placing process at a warehouse section. Inbound areas and the outbound gates provide enough space, therefore no blocking situation is modeled. The time a forklift is blocked by the action of other forklifts is recorded separately during simulation runs which allows detailed analyses afterwards.

Organizational data contains the process structure of the system, aspects of staff organization and resource allocation rules. One main aspect is to organize the fleet of forklifts sufficiently to handle the daily pallet volume. Therefore, a module to take the detailed shift schedule for each day into account has been implemented into the model. Additionally, each forklift is allocated to a specific team to consider the staff organization within the real warehouse. In the basic scenario there are three teams, each one is dedicated to specific task types which occur in the warehouse:

- inbound team for placing the incoming goods into the warehouse

- outbound team for picking the outgoing goods and transport them to the destination buffers and to the order-picking area

- value-added team for transporting materials to the value-added area and placing finished goods into the warehouse

However, the implementation allows other team configurations based on the task types which are also used in the simulation study for alternative scenarios. For the basic scenario a simple forklift assignment within the teams is used: if a task occurs and more than one forklift is free, the forklift is chosen randomly. The rules to assign a specific task for a freed forklift are described within the following paragraphs as task assignment strategies.

The other main aspect of warehouse organization are the assignment strategies considering the warehouse itself. For incoming goods the following strategies are necessary:

- The storage location assignment strategy decides on the specific storage location of an incoming pallet. To create a realistic model the rules of the real warehouse management software have been implemented which base on a search algorithm considering the main characteristics of the pallets, such as item type, storage category and height, in combination with the characteristics of the warehouse locations. The implementation is therefore flexible to adapt to various setups of warehouse management by changing the characteristics of the warehouses and the incoming pallets correspondingly.

- The task assignment strategy decides on the operating order of the inbound tasks. For the simulation study, the typically used first-come-first-served (FCFS) strategy has been implemented.

Furthermore, the following strategies for the outgoing goods are considered for the basic scenario in the simulation model of the warehouse:

- The selection strategy for outgoing pallets is based on the article type, the date of expiry of a specific pallet and the type of pallet storage (single or double stacked pallets).

- The task assignment strategy decides on the operating order of the outbound tasks. The specific rules of the real warehouse management system have been implemented into the simulation model with the help of a priority indicator. Highest priority is given to replenishment tasks for the order-picking area. The priority of normal outbound tasks rises as the cut-off time of the outbound order is approaching.

System load data includes two categories of pallet volumes. Firstly, the initial status of the warehouse has to be taken into account due to the fact that the filling level has significant impact on the handling performance. Secondly, the handled volumes for one typical week have to be determined to get a reliable statement on the system performance under various operating scenarios. After the determination of an appropriate week both data categories have been derived from real operational data of the 


\section{Clausen, Dabidian, Diekmann, Goedicke, and Pöting}

warehouse management system. For the initial status a list of the warehouse storage locations and their status at the beginning of the considered week has been investigated and a dedicated simulation object has been implemented which creates the pallets with their characteristics in the predefined locations.

Furthermore, all handling tasks for inbound, outbound, replenishment of order-picking and servicing the value-added-area have been taken from the real system and prepared to integrate them into the simulation model. In addition to the typical pallet characteristics, the time when the task is dispatched to the system is a very important information which has been considered. Within the simulation model, specific objects are implemented to create the tasks at the predefined time.

To validate the basic scenario and compare different alternative scenarios the system performance has to be measured within the simulation. Therefore, different results are recorded during the simulation runs and aggregated into key figures and diagrams afterwards. To measure the handling performance all relevant time stamps of each operating cycle described in section 2 are saved in a table and analyzed in a standardized way for the different teams after each simulation run. Additionally, the stock of unfinished transport tasks is recorded every five minutes as well as the amount of buffered pallets in the different inbound zones. In the following section the experiments and results of the simulation study are explained.

\section{EXPERIMENTS AND RESULTS}

For the simulation study, a representative week in terms of system volume has been chosen. This week contains five days with a operating time of 16 hours per day. Start and end time as well as the team strength depends on shift-planning of the team leader in the considered week. In a first step, the modeling of the basic as-is-scenario has to be validated.

\subsection{Validation Scenario}

In this scenario, the model has been validated on the basis of the process study of the warehouse operating company which also served as source for the handling parameters, except the transport times. In accordance to Figure 2 the resulting average processing times of each activity are calculated from the simulation runs. Afterwards, the total process time of both methods, the process study and the simulation model could be compared as demonstrated in Figure 3 to ensure the correctness of the model. Additionally, the physical parameters for the forklifts were tested by measuring reference routes, which were collected in the real system to set up the process study. Both approaches confirm that the developed simulation model properly represents the real system to conduct further scenarios for improving system performance.

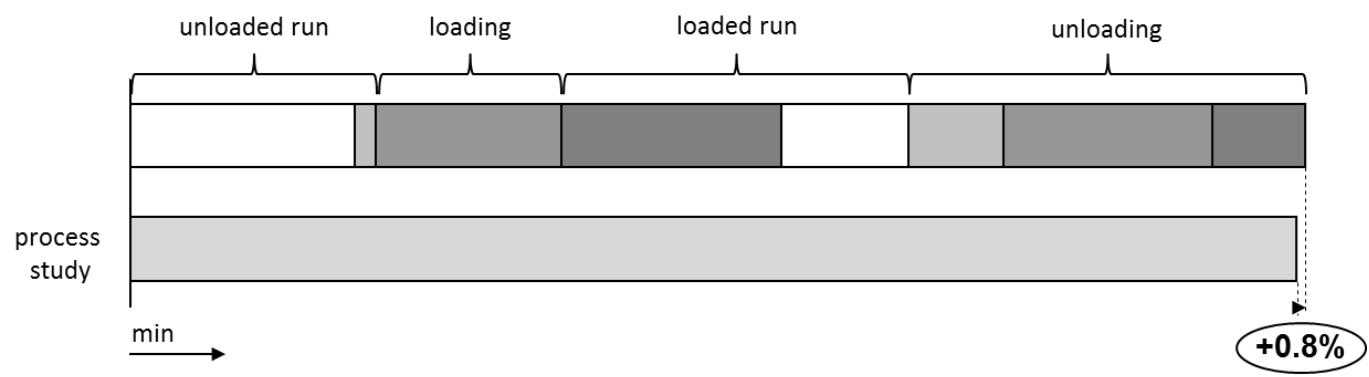

Figure 3: Comparison of the average operational cycle (inbound team).

Subsequent to the successful validation, the experimental plan for the simulation study was set up which contains two different scenarios to improve the system on three different levels as illustrated in Figure 4. Handling parameters for loading and unloading times are considered to be fixed during the simulation study. 
Clausen, Dabidian, Diekmann, Goedicke, and Pöting

\begin{tabular}{|c|c|c|}
\hline \multirow{6}{*}{ Scenario 1} & \multicolumn{2}{|r|}{ Level 1: Storage Allocation } \\
\hline & Measure: & New Storage-Rag Profile / Zones \\
\hline & Influence: & $>$ Loaded Run \\
\hline & \multicolumn{2}{|r|}{ Level 2: Storage Allocation } \\
\hline & Measure: & Alternating Aisles \\
\hline & Influence: & $>$ Blocking \\
\hline \multirow{3}{*}{ Scenario 2} & \multicolumn{2}{|r|}{ Level 3: Fleet Control } \\
\hline & Measure: & Double Cycle \\
\hline & Influence: & $>$ Unloaded Run \\
\hline
\end{tabular}

Figure 4: Experimental plan of the simulation study.

\subsection{Scenario 1: New Storage Allocation Strategy}

The first scenario examines the impact of an optimized storage allocation on the operating cycle. Specific measures taken were the implementation of a new item-type classification combined with a new zone allocation, considering the driving routes lengthwise through the warehouse, as well as an optimized storage-rag profile considering the item's ABC-classification to minimize routes into the aisles. The main focus was to get shorter overall loaded runtimes by an intelligent allocation and management of storage locations.

In addition, a new algorithm has been implemented into the warehouse management system which enhances the storage-bay allocation to alternate the chosen aisles for received goods. Consecutive inbound tasks of the same item-type (zones, ABC-class) are routed into alternating aisles to prevent blocking of forklifts that are assigned to similar tasks in quick succession. Thus, the implemented improvements in this scenario try to minimize the overall time for loaded runs as well as the time when forklifts are blocked.

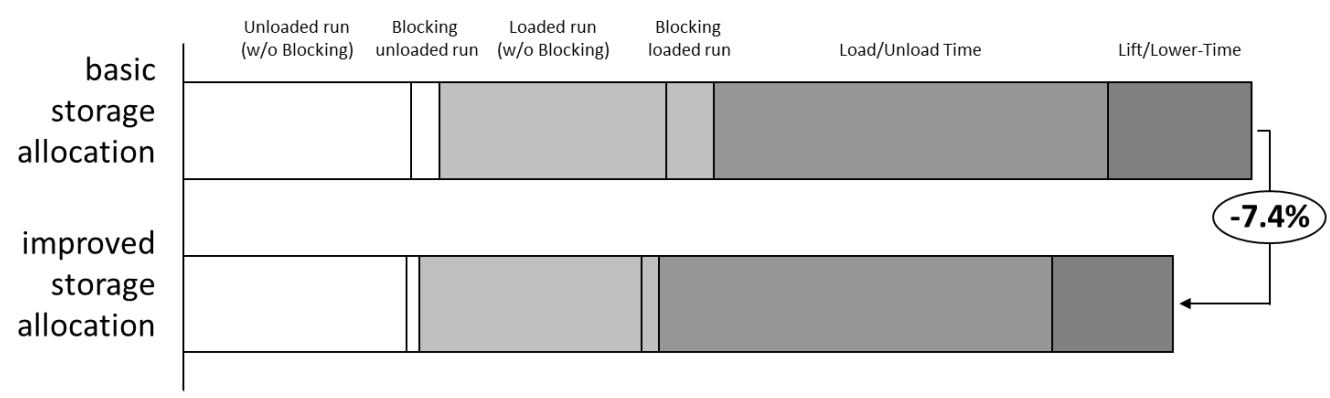

Figure 5: Average task execution time comparison for basic scenario and scenario 1.

The resulting average times for transport task execution are compared for the basic scenario and the scenario 1 with improved storage allocation in Figure 5. The activities for loading and unloading do not change because they are not influenced by storage location assignment. However, a significant decrease in blocking times by $57 \%$ for unloaded runs and $64 \%$ for loaded runs improves the handling efficiency in the warehouse. Only little decrease of approx. $2 \%$ could be achieved for the unloaded run as well as the loaded run itself due to the fact that the changes in storage allocation improved the transport distances for a number of tasks but also had a negative effect on other ones. This consequence could only be prevented by an additional new arrangement for outbound buffers. Furthermore, the new rules for storage assignment induce $17 \%$ less time for lifting and lowering. In sum the measures to improve system performance result in a decrease of average execution time for a transport task by $7,4 \%$. 


\subsection{Scenario 2: New Fleet Control}

\section{Clausen, Dabidian, Diekmann, Goedicke, and Pöting}

In the second scenario, the controlling of the different teams has been redesigned. The focus in the scenario was to minimize the unloaded runtime by implementing a double cycle system. Therefore, the inbound team and the outbound team are merged into one group with a common transport task pool, while the value-added team stays independent due to its specific tasks. Additionally, an intelligent and variable control system for allocating the transport tasks has been developed. It assigns the next transport task mainly based on the shortest distance of the unloaded run out of three task types, inbound as well as outbound and replenishment. To prevent delays of outbound tasks, a dynamic cut-off rule was implemented to give a higher priority to transport tasks which have their cut-off time during the next two hours.

In order to ensure the comparability, the amount and time-schedule of the existing forklifts in the model has been the same as in the validation scenario. The results are illustrated in Figure 6 and can be summarized as follows.

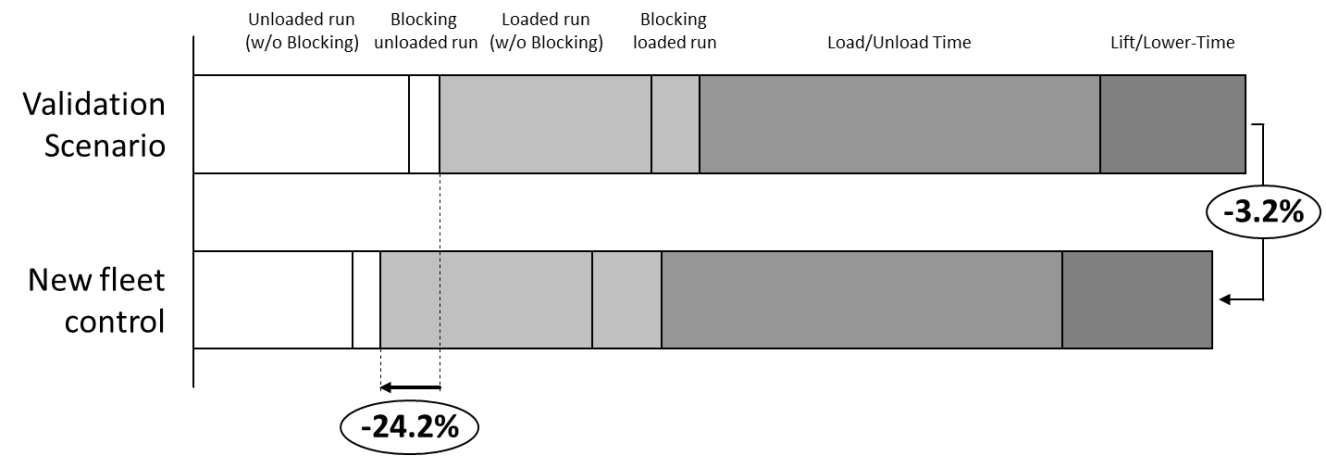

Figure 6: Average task execution time comparison for the basic scenario and scenario 2.

Implementing a central dispatching of transport tasks which allows efficient double cycles can significantly decrease the time of unloaded runs by $24.2 \%$. However, the blocked time during loaded runs increases slightly due to the fact that more forklifts are available for the pool of transport tasks. Therefore, the probability to enter the same aisle is higher because the storage allocation of the basic scenario is used in both cases. The process time for all other activities does not change as they are not affected by the task dispatching. To sum up, the improvement on the average total process time is only $3.2 \%$. However, the development of the stock of available transport tasks has to be evaluated as well, to show one main influence of the new dispatching strategy on system performance. As illustrated in Figure 7, the availability of one big team for executing the transport tasks creates potential to reduce the overall teamsize by a greater flexibility.
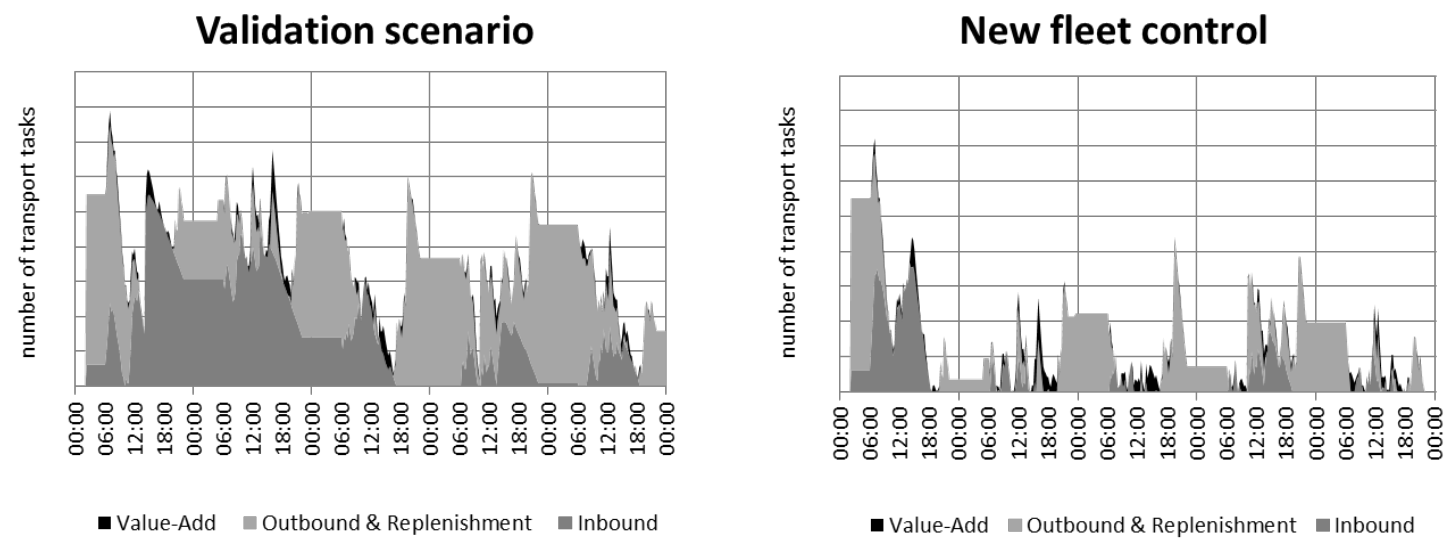

Figure 7: Development of stock of available transport tasks for the basic scenario and scenario 2. 


\section{CONCLUSION}

Clausen, Dabidian, Diekmann, Goedicke, and Pöting

The paper shows a successful application of simulating a manually operated distribution warehouse which is very difficult to improve by static methods due to its complex and dynamic processes. A microscopic model of a large warehouse has been implemented and validated based on a real system which allows the analysis of different operating strategies. The evaluation of strategies to improve warehouse operations on three main levels shows significant potential to improve handling performance. In a first step, the adjustment of the storage-rag profile and the usage of alternating aisles can decrease the average process time by approx. 7\%. The implementation of the new strategy is highly recommended as a first optimization step, based on detailed analysis of the structure of items handled in the warehouse (ABCclassification, zones). Additionally, the sequential implementation of a new holistic dispatching of transport tasks for inbound and outbound offers huge potential of $24 \%$ to decrease the unloaded travel time within the warehouse and gives a higher flexibility to adjust the forklift capacity to the actual stock of transport tasks. In order to avoid additional blocking, caused by the bigger forklift team, it is important to implement a storage location assignment strategy that uses alternating aisle locations in advance. Due to the fact that the implementation of a new dispatching algorithm requires some additional invest into the warehouse management software, it is recommended for large warehouses which will significantly benefit from the increased flexibility in capacity adjustment.

\section{REFERENCES}

Chan, F.T.S. 2002. "Design of material handling equipment selection system: an integration of expert system with analytic hierarchy process approach." Integrated Manufacturing Systems 13(1):58-68.

Clausen, U., P. Dabidian, D. Diekmann, I. Goedicke, and J. Kaffka. 2012. "Optimizing LTL terminal performance by simulation" In Proceedings of the European Simulation and Modelling Conference 2012, 386-393.

de Koster, R., T. Le-Anh, and R. van der Meer. 2004. "Testing and classifying vehicle dispatching rules in three real-world settings." Journal of Operations Management 22: 369-386.

de Koster, R., T. Le-Duc, and K. J. Roodbergen. 2007. "Design and control of warehouse order picking: A literature review." European Journal of Operational Research 182(2): 481-501

Deymann S., and L. Neumann. 2008. "TRANSSIMNODE - a simulation tool for logistics nodes" In Proceedings of the Industrial Simulation Conference 2008, 283-287.

Egbelu, P. J., and J. M. A. Tanchoco. 1984. "Characterization of automated guided vehicle dispatching rules" International Journal of Production Research 22:359-374.

Furmans, K., C. Huber, and J. Wisser. 2009. "Queuing models for manual order picking systems with blocking" Logistics Journal 01:1-16.

Gu, J., M. Goetschalckx, and L. McGinnis. 2007. "Research on warehouse operation: A comprehensive review" European Journal of Operations Research 177(1): 1-21

Kille, C., and M. Schwemmer. 2012. Die TOP 100 der Logistik. Marktgrößen, Marktsegmente und Marktführer. Hamburg: DVV-Media Group.

Klein, C. M. and J. Kim. 1996. “AGV dispatching” International Journal of Production Research, 34(1): 95-110.

Koo, P.-H., and J. Jang. 2002. "Vehicle travel time models for AGV systems under various dispatching rules" The International Journal of Flexible Manufacturing Systems 14(3):249 - 261.

Le-Anh, T., and R. De Koster. 2006. "A review of design and control of automated guided vehicle systems" European Journal of Operations Research 171:1-23.

Petersen, C., and G. Aase. 2004. "Improving order-picking performance through the implementation of class-based storage" International Journal of Physical Distribution \& Logistics Management 34(7): 534-544. 
Clausen, Dabidian, Diekmann, Goedicke, and Pöting

Takakuwa, S., H. Takizawa, I. Kumiko, and S. Hiraoka. 2000. "Simulation of distribution systems: simulation and analysis of non-automated distribution warehouses" In Proceedings of the 32nd conference on Winter simulation 2000, 1177-1184.

Transport Intelligence Ltd. 2012. "Transport intelligence: market intelligence for the transport and logistics industry". In Global Contract Logistics 2012. United Kingdom.

VDI Guideline 3633 Part 1. 2010. Simulation of systems in materials handling, logistics and productionPart 1 Fundamentals. Edited by The Association of German Engineers. Duesseldorf.

\section{AUTHOR BIOGRAPHIES}

PROF DR.-ING UWE CLAUSEN is the managing director of the Institute of Transport Logistics at the TU Dortmund University and director of the Fraunhofer-Institute for Material Flow and Logistics (IML). He studied computer sciences at the University of Karlsruhe (TH) and finished his doctoral thesis on transportation network optimization at TU Dortmund University in 1995. Afterwards, he worked at the "Deutsche Post AG" and joined Amazon.de in 1999. As operations director he was responsible for setting up the new Amazon Logistics Center in Bad Hersfeld. In 2001 Uwe Clausen accepted the chair "Transport Systems and Logistics" at TU Dortmund University, which became the Institute of Transport Logistics in 2011. He is amongst others chairman of EffizienzCluster LogistikRuhr and a member of ECTRI European Conference of Transport Research Institutes and of the Scientific Advisory Board of German Logistics Association (BVL). His email address is clausen@itl.tu-dortmund.de and his web page is http://www.itl.tu-dortmund.de/cms/de/home/team/mitarbeiter/Clausen_Uwe.html.

PEIMAN DABIDIAN attended TU Dortmund University, where he studied computer science with business studies as a minor subject. He obtained his degree in 2010. Afterwards, he worked at paluno the Ruhr Institute for Software Technology at University of Duisburg-Essen and joined the Institute of Transport Logistics at TU Dortmund University in 2012. He works as a scientific assistant and as a fulltime $\mathrm{PhD}$ student in the field of resource utilization in LTL terminals and simulation in traffic and logistics. His email address is dabidian@itl.tu-dortmund.de.

DANIEL DIEKMANN attended TU Dortmund University, where he studied logistics and obtained his degree in 2010. He works at the Institute of Transport Logistics at TU Dortmund University as a scientific assistant and as a full-time PhD student, doing research in the fields of LTL and CEP terminals as well as simulation in traffic and transport logistics. His email address is diekmann@itl.tu-dortmund.de.

INA GOEDICKE is a scientific assistant and full time $\mathrm{PhD}$ student at the Institute of Transport Logistics. She studied logistics at TU Dortmund University and obtained her degree in 2009. Since 2011 she is the team leader of the institute's research group on simulation and logistics engineering. She does research in the fields of logistic terminal layout, yard management strategies and simulation in traffic and transport logistics. Her email address is goedicke@itl.tu-dortmund.de.

MORITZ PÖTING attended TU Dortmund University, where he received his B. Sc. in Logistics in 2012. He works as student assistant and M. Sc. student at the Institute of Transport Logistics in simulation projects on traffic and transport logistics. His email address is poeting@itl.tu-dortmund.de. 